\title{
The utility of noninvasive scores in non-alcoholic fatty liver disease patients with normal and elevated serum transaminases
}

\author{
Eda Kaya ${ }^{1}$, (10) Alev Bakir2, (10) Fatih Eren ${ }^{3,4}$, (ㄱ) Yusuf Yilmaz ${ }^{4,5}$ \\ ${ }^{1}$ Department of Internal Medicine, Helios Hospital Schleswig, Academical Educational Hospital of Luebeck and Kiel Universities, Schleswig, Germany; \\ ${ }^{2}$ Department of Biostatics and Medical Informatics, Halic University Faculty of Medicine, Istanbul, Turkey; ${ }^{3}$ Department of Medical Biology, Marmara University \\ Faculty of Medicine, Istanbul, Turkey; ${ }^{4}$ Institute of Gastroenterology, Marmara University, Istanbul, Turkey; ${ }^{5}$ Department of Gastroenterology, \\ Marmara University Faculty of Medicine, Istanbul, Turkey
}

\begin{abstract}
Background and Aim: Noninvasive scores are developed for the estimation of advanced fibrosis, including parameters in addition to transaminases in non-alcoholic fatty liver disease (NAFLD). In this study, we aimed to investigate the diagnostic performances of Fibrosis-4 (FIB-4) and NAFLD Fibrosis Score (NFS) in the estimation of advanced fibrosis comparing patients with normal and elevated transaminases.

Material and Methods: We retrospectively analyzed the prospectively collected data of a total of 407 consecutive patients with biopsy-proven NAFLD. FIB-4 scores of $<1.3$ and $>2.67$ or $<1.45$ and $>3.25$ indicated a low and high risk for advanced fibrosis, respectively. NFS scores of $<-1.455$ and $>0.676$ were used to assess low and high risk for advanced fibrosis, respectiv.

Results: FIB- 4 cutoffs of $<1.3$ and $<1.45$ for low risk of advanced fibrosis had a sensitivity of $70 \%$ and $54 \%$ in patients with elevated transaminases and $70 \%$ and $52 \%$ in patients with normal transaminases, respectively. The specificities for the cutoffs of $>2.67$ and $>3.25$ were $97 \%$ and $98 \%$ in patients with elevated transaminases and $99 \%$ and $100 \%$ in patients with normal transaminases, respectively. Concerning NFS, we found similar results.

Conclusion: FIB-4 and NFS showed acceptable diagnostic performance in the exclusion of advanced fibrosis in both populations with normal and elevated transaminases.
\end{abstract}

Keywords: Biopsy; liver fibrosis; non-alcoholic fatty liver disease; sensitivity and specificity.

\section{Introduction}

Non-alcoholic fatty liver disease (NAFLD) is currently the most prevalent chronic liver disease worldwide. NAFLD is estimated to affect 25\% of the world population. ${ }^{[1]}$ Non-alcoholic steatohepatitis (NASH), the subtype of NAFLD, is already among the top etiologies for hepatocellular carcinoma. Due to the close association of NAFLD with obesity and type 2 diabetes mellitus, an increase in incidence and prevalence of

Received: December 21, 2019; Accepted: January 07, 2020; Available online: January 20, 2020

Corresponding author: Yusuf Yilmaz; Marmara Universitesi, Gastroenteroloji Enstitusu, P.K. 53, Basibuyuk, Maltepe, 34840, Istanbul, Turkey

Phone: +90 21642143 86; e-mail: dryusufyilmaz@gmail.com

(c) Copyright 2020 by Hepatology Forum - Available online at www.hepatologyforum.org
NAFLD is predicted in the recent models. In this respect, NAFLD represents a significant economic and public health burden for the world. ${ }^{[2]}$ NAFLD is characterized by evidence of hepatic steatosis and lack of secondary causes of hepatic fat accumulation, such as significant alcohol consumption, long-term use of a steatogenic medication, or hereditary disorder. NAFLD exists on a spectrum from simple steatosis to NASH. ${ }^{[3-}$ ${ }^{5]}$ The severity of hepatic fibrosis plays especially a significant role in the determination of NAFLD prognosis. NAFLD patients with advanced fibrosis have an increased tendency to progress to cirrhosis, hepatocellular carcinoma, end-stage liver failure and even liver-related mortality, which underlines the importance of timely detection of advanced fibrosis. ${ }^{[6,7]}$

To date, liver biopsy remains the reference standard for detecting inflammation and staging fibrosis, which are more likely to be associated with liver-related morbidity and mortality. However, liver biopsy has various main limitations, including high cost, its invasive nature, risk of complications and small size of harvested liver tissue, which may not represent the disease status. ${ }^{[8,9]}$ Therefore, there is a significant effort to replace liver biopsy with non-invasive modalities, such as clinical and blood-based biochemical testing approaches. ${ }^{[9,10]}$ Among those, in the recent NAFLD guidelines, Fibrosis-4 Index (FIB-4) and NAFLD Fibrosis Score (NFS) were recommended to use in the estimation of advanced fibrosis. ${ }^{[3,9]}$

Clinically, the most common pattern of NAFLD is mildly elevated liver transaminases, although elevated serum transaminase levels poorly correlate with liver histology. ${ }^{[1]}$ However, serum transaminases are also included in FIB- $4^{[12]}$ and NFS ${ }^{[13]}$ as a part of the calculation although there is a lack of evidence whether their diagnostic accuracy differs according to serum transaminase levels. In this study, we aimed to investigate the diagnostic performances FIB-4 and NFS in the identification of advanced fibrosis comparing biopsy-proven NAFLD patients with normal and elevated serum transaminases. We also sought to find optimal cutoffs for our study patients with and without elevated liver enzymes, respectively.

\section{Materials and Methods \\ Patients}

This is a retrospective analysis of the prospectively collected data. A total of 407 consecutive biopsy-proven adults ( $>18$ years) as NAFLD patients who were followed up in outpatient facilities of the Marmara University School of Medicine were recruited for this study. According to our laboratory, an alanine transaminase (ALT) level of $\leq 40 \mathrm{U} / \mathrm{L}$ and an 
Table 1. General characteristics of the study patients $(n=407)$

Patients with normal
liver enzymes
ALT $\leq 40 \mathrm{U} / \mathrm{L}$ and
AST $\leq 37 \mathrm{U} / \mathrm{L}(\mathrm{n}=107)$

Age, median [min.-max.], year

Gender, male/female ( $\mathrm{n}$ )

BMI, median [min.-max.], $\mathrm{kg} / \mathrm{m}^{2}$

Lean/overweight/obese ( $\mathrm{n}$ )

Metabolic syndrome (yes/no)

Type 2 diabetes mellitus (yes/no)

Hypertension (yes/no)

Hyperlipidaemia (yes/no)

Waist circumference, median [min.-max.], cm

Hip circumference, median [min.-max.], $\mathrm{cm}$

Albumin, median [min.-max.], mg/dL

AST, median [min.-max.], U/l

ALT, median [min.-max.], U/I

ALP, median [min.-max.], U/I

GGT, median [min.-max.], U/I

LDH, median [min.-max.], U/L

Total bilirubin, median [min.-max.], mg/dL

Direct bilirubin, median [min.-max.], mg/dL

Total protein, median [min.-max.], g/dL

Total cholesterol, median [min.-max.], mg/dL

Triglycerides, median [min.-max.], mg/dL

HDL cholesterol, median [min.-max.], mg/dL

LDL cholesterol, median [min.-max.], mg/dL

Leucocytes, median [min.-max.], per $\mathrm{mL}$

Platelets, median [min.-max.], $\times 10^{3}$ per microliter

Haemoglobin, median [min.-max.], mg/dL

Glucose, median [min.-max.], mg/dL

Creatinine, median [min.-max.], mg/dL

BUN, median [min.-max.], mg/dL

Uric acid, median [min.-max.], mg/dL

$\mathrm{HbA1c}$, median [min.-max.], \%

HOMA-IR, median [min.-max.]

\begin{tabular}{|c|c|c|c|}
\hline 52 [29-71] & 50 [21-71] & 50 [21-71] & $0.095^{\mathrm{a}}$ \\
\hline $38 / 69$ & $130 / 170$ & $168 / 239$ & $0.158^{b}$ \\
\hline 32.1 [23.3-56.0] & 31.6 [21.3-52.0] & 31.9 [21.4-56.0] & $0.112^{\mathrm{a}}$ \\
\hline $5 / 28 / 74$ & $12 / 84 / 204$ & $17 / 112 / 278$ & $0.907^{b}$ \\
\hline $79 / 28$ & $211 / 89$ & $290 / 117$ & $0.492^{b}$ \\
\hline $54 / 53$ & $137 / 163$ & $191 / 216$ & $0.393^{b}$ \\
\hline $49 / 58$ & $121 / 179$ & $170 / 237$ & $0.325^{b}$ \\
\hline $75 / 32$ & $182 / 118$ & $257 / 150$ & $0.083^{b}$ \\
\hline 105 [81-147] & 104 [79-146] & 105 [79-147] & $0.117^{a}$ \\
\hline 112 [92-155] & 109 [84-144] & 110 [84-155] & $0.024^{a}$ \\
\hline $4.6[3.5-5.3]$ & $4.6[3.4-5.6]$ & $4.6[3.4-5.6]$ & $0.330^{\mathrm{a}}$ \\
\hline 25 [11-37] & 47 [22-302] & 40 [11-302] & $<0.001^{a}$ \\
\hline 29 [12-39] & 70 [18-343] & 59 [12-343] & $<0.001^{a}$ \\
\hline 82 [34-485] & 94 [25-625] & 92 [25-625] & $0.036^{a}$ \\
\hline 35 [9-342] & 54 [10-559] & 49 [9-559] & $<0.001^{a}$ \\
\hline 206 [132-732] & 229 [19-969] & 221 [19-969] & $0.003^{a}$ \\
\hline $0.63[0.21-1.82]$ & $0.66[0.12-6.10]$ & $0.65[0.12-6.10]$ & $0.096^{a}$ \\
\hline $0.13[0.01-0.54]$ & $0.18[0.01-1.20]$ & $0.16[0.01-1.20]$ & $<0.001^{a}$ \\
\hline 7.7 [6.5-9.0] & 7.8 [6.5-9.1] & $7.8[6.5-9.1]$ & $0.273^{a}$ \\
\hline 211 [79-417] & 210 [74-419] & 210 [74-419] & $0.457^{a}$ \\
\hline 170 [38-1107] & 162 [37-584] & 163 [37-1107] & $0.498^{a}$ \\
\hline 44 [26-91] & 45 [25-96] & 45 [25-96] & $0.872^{\mathrm{a}}$ \\
\hline 127 [35-265] & 134 [28-400] & 132 [28-400] & $0.202^{a}$ \\
\hline 7000 [2400-14700] & 6960 [3200-14900] & 6970 [2400-14900] & $0.184^{a}$ \\
\hline 225 [77-475] & 230 [89-435] & 229 [77-475] & $0.665^{a}$ \\
\hline $13.8[9.1-17.5]$ & $14.3[8.5-18.9]$ & $14.1[8.5-18.9]$ & $0.004^{a}$ \\
\hline 105 [70-240] & 104 [71-307] & 105 [70-307] & $0.850^{a}$ \\
\hline $0.71[0.45-1.12]$ & $0.76[0.41-2.13]$ & $0.75[0.41-2.13]$ & $0.108^{a}$ \\
\hline 14 [6-29] & $15[7-36]$ & 14 [6-36] & $0.367^{a}$ \\
\hline 6 [2.1-8.2] & $6.4[0.7-11.1]$ & $6.2[0.7-11.1]$ & $0.184^{a}$ \\
\hline 5.9 [4.5-8.9] & $5.9[4.0-11.1]$ & $5.9[4.0-11.1]$ & $0.555^{\mathrm{a}}$ \\
\hline $4.65[1.06-15.53]$ & $5.29[1.0-28.76]$ & $4.90[1.0-28.76]$ & $0.084^{a}$ \\
\hline
\end{tabular}

ALT: Alanine aminotransferase; AST: Aspartate aminotransferase; BMI: Body mass index; ALP: Alkaline phosphatase; GGT: Gama glutamyl transferase; LDH: Lactate dehydrogenase; HDL: High density lipoprotein; LDL: Low density lipoprotein; SD: Standard deviation; BUN: Blood urea nitrogen; HbA1c: HemoglobinA1c; HOMA-IR: Homeostatic model assessment for insulin resistance; Min.: Minimum; Max. Maximum. Statistical tests, a: Mann-Whitney U Test, b: Chi-Square Test.

$158^{b}$

$.907^{\mathrm{b}}$

$393^{\mathrm{b}}$

$0.325^{\mathrm{b}}$

$0.117^{\mathrm{a}}$

$330^{\mathrm{a}}$

$0.001^{\mathrm{a}}$

$0.001^{\mathrm{a}}$

$0.001^{\mathrm{a}}$

$.003^{a}$

$0.001^{a}$

$273^{\mathrm{a}}$

$.498^{a}$

$872^{a}$

$184^{a}$

$665^{a}$

$.850^{a}$

$108^{a}$

$184^{a}$

$555^{a}$ aspartate transaminase (AST) level of $\leq 37 \mathrm{U} / \mathrm{L}$ were defined as normal. An ALT level of $>40 \mathrm{U} / \mathrm{L}$ and/or AST level of $>37 \mathrm{U} / \mathrm{L}$ were considered elevated serum transaminase levels. Exclusion criteria were explained in detail previously. ${ }^{[14]}$ The groups were well-matched concerning age, gender and body mass index (BMI). Liver biopsies were evaluated by a single pathologist expertized in the liver as previously described ${ }^{[15]}$ and a histological fibrosis score $\mathrm{F} \geq 3$ was used to define advanced fibrosis. ${ }^{[16]}$ The pathologist was blinded to FIB-4 and NFS results.

\section{Calculation of the FIB-4 and NFS}

FIB-4 scores were calculated using four parameters (platelet count,

\section{Patients with elevated \\ liver enzymes \\ ALT $>40 \mathrm{U} / \mathrm{L}$ and/or AST $>37 \mathrm{U} / \mathrm{L}(\mathrm{n}=300)$}

\section{Total patients}

$(n=407)$

p 
Table 2. Histological characteristics of the study patients

\begin{tabular}{|c|c|c|c|c|}
\hline & $\begin{array}{l}\text { Patients with } \\
\text { normal liver } \\
\text { enzymes } \\
\text { ALT } \leq 40 \mathrm{U} / \mathrm{L} \text { and } \\
\text { AST } \leq 37 \mathrm{U} / \mathrm{L} \\
(\mathrm{n}=107)\end{array}$ & $\begin{array}{l}\text { Patients with } \\
\text { elevated liver } \\
\text { enzymes } \\
\text { ALT>40 U/L and/or } \\
\text { AST>37 U/L } \\
(n=300)\end{array}$ & $\begin{array}{l}\text { Total } \\
\text { patients } \\
(n=407)\end{array}$ & $\mathbf{p}$ \\
\hline NAS score (NASH CRN), median [min.-max.] & $4[1-8]$ & $6[1-8]$ & $5[1-8]$ & $<0.001^{\mathrm{a}}$ \\
\hline SAF algorithm classification, NASH/NAFL, (n) & $92 / 15$ & $280 / 20$ & $372 / 35$ & $0.033^{b}$ \\
\hline Grade of steatosis (S) according to the SAF score: S0/S1/S2/S3 (n) & $0 / 47 / 41 / 19$ & $0 / 58 / 112 / 130$ & $0 / 105 / 153 / 149$ & $<0.001^{b}$ \\
\hline Stage of activity $(A)$ according to the SAF score: $A 0 / A 1 / A 2 / A 3 / A 4,(n)$ & $3 / 11 / 36 / 32 / 25$ & $3 / 14 / 55 / 100 / 128$ & $6 / 25 / 91 / 132 / 153$ & $<0.001^{\text {b }}$ \\
\hline Stage of fibrosis $(F)$ according to the SAF score: F0/F1/F2/F3/F4, (n) & $48 / 22 / 14 / 16 / 7$ & $58 / 111 / 64 / 51 / 16$ & $106 / 133 / 78 / 67 / 23$ & $<0.001^{b}$ \\
\hline Mild disease/severe disease (n) & $13 / 94$ & $16 / 284$ & 29/378 & $0.033^{b}$ \\
\hline Significant fibrosis $(F \geq 2)$, (n) & 37 & 131 & 168 & $0.101^{b}$ \\
\hline Advanced fibrosis $(F \geq 3),(n)$ & 23 & 67 & 90 & $0.858^{b}$ \\
\hline Cirrhosis $(F=4),(n)$ & 7 & 16 & 23 & $0.642^{b}$ \\
\hline
\end{tabular}

NASH: Non-alcoholic steatohepatitis; NAFL: Non-alcoholic fatty liver; NAS: Non-alcoholic fatty liver disease activity score; CRN: Clinical Research Network; Min.:

Minimum; Max. Maximum. Statistical tests, a: Mann-Whitney U Test, b: Chi-Square Test.

pressed as mean \pm standard deviation and compared with the Independent Sample t-test. Nonnormally distributed continuous data were expressed as median [minimum-maximum] and compared using the Mann-Whitney $\mathrm{U}$ test. Categorical data were given as count and percentages and analyzed using the chi-square test. Receiver operating characteristics (ROC) analysis was carried out to calculate the area under the curve to identify the optimal cutoff value for FIB-4 and NFS. The highest value for Youden's index was defined as the optimal cutoff. The sensitivity, specificity, positive predictive value, and negative predictive value for each test were also presented. Cohen's kappa was used as a measure of agreement between the noninvasive test and the reference standard. All analyses were performed using the SPSS 24.0 statistical package (IBM, Armonk, NY, USA). A two-tailed $p$ value $<0.05$ was considered statistically significant.

\section{Ethics}

This study was conducted in adherence to the Helsinki Declaration and was approved by the Marmara University ethics committee. Due to the retrospective nature of this study, the need for informed consent was waived. This work has been supported Marmara University Scientific Research Projects Coordination Unit under grant number: SAG-CTUP-150218-0033.

\section{Results}

From 407 biopsy-proven NAFLD patients, 107 patients $(26.3 \%)$ had normal and 300 patients $(73.7 \%)$ elevated serum transaminases. General characteristics of the study patients were presented in Table 1, comparing patients with normal and elevated serum transaminase levels. In Table 2, the histological characteristics of the patients were summarized. Among patients with normal serum transaminases, 23 patients $(21.5 \%)$ had advanced fibrosis, whereas among patients with elevated serum transaminases were 67 (22.3\%). The prevalence of advanced fibrosis among those groups did not significantly differ $(\mathrm{p}=0.858)$.

In patients with normal serum transaminase levels, using the previously published cutoff values for FIB-4 $(<1.3$ and $<1.45$ for low risk of advanced fibrosis and $>2.67$ and $>3.25$ for high risk of advanced fibrosis), we classified $73(68.2 \%)$ and $79(73.8 \%)$ patients as low risk, $30(28.0 \%)$ and $27(25.2 \%)$ patients as indeterminate risk and $4(3.7 \%)$ and $1(0.9 \%)$ patients as high risk of advanced fibrosis, respectively. Considering NFS, 41 patients $(38.3 \%)$ were classified as low risk, 52 $(48.6 \%)$ as indeterminate and $14(13.1 \%)$ as high risk for advanced fibrosis. Using the cutoff values for FIB-4 $(<1.3$ and $<1.45$ for low risk of advanced fibrosis and $>2.67$ and $>3.25$ for high risk of advanced fibrosis) in patients with elevated serum transaminases, we identified $171(57 \%)$ and 210 patients $(70 \%)$ as low risk, $111(37 \%)$ and 75 patients $(25 \%)$ as indeterminate risk and $18(6 \%)$ and 15 patients $(5 \%)$ as high risk for advanced fibrosis, respectively. For NFS, we classified 159 patients (53\%) as low, $127(42.3 \%)$ as indeterminate and $14(4.7 \%)$ as high risk of advanced fibrosis.

The diagnostic performances of FIB-4 and NFS in patients with normal and elevated serum transaminases were presented in Table 3 and Table 4, respectively. Accordingly, especially cutoffs indicating a high risk of advanced fibrosis had a relatively good diagnostic performance in exclusion of advanced fibrosis considering the high specificity values for both of the groups, including all the cutoffs of FIB-4 and NFS. However, low sensitivity values indicated low diagnostic performance in the identification of advanced fibrosis. Concerning optimal cutoff values, we detected the best cutoff values at $>1.2$ and $>1.3$ for FIB- 4 for elevated and normal transaminase levels, whereas for NFS at $>-1.483$ and $>-0.587$, respectively. We found overall suboptimal agreement with the noninvasive tests and reference standard considering low kappa values both for the cutoffs recommended in the literature and optimal cutoffs revealed in our study.

\section{Discussion}

In this study, to our knowledge, we compared for the first time the diagnostic performances of FIB-4 and NFS using different cutoff values recommended in the literature between patients with normal and elevated serum transaminase levels, and we found similar diagnostic performance in both of the groups. The optimal FIB-4 cutoff value revealed in our population for patients with normal serum transaminases was $>1.3$ for the identification of advanced fibrosis, which was coherent 
Table 3. Diagnostic performance of the FIB-4 in patients with normal $(n=107)$ and elevated $(n=300)$ serum transaminases in assessment of advanced fibrosis

$\begin{array}{lllllllllll}\text { Sensitivity (\%) } & \text { Specificity (\%) } & \text { AUC } & 95 \% \mathrm{Cl} & \text { FN } & \text { FP } & \text { PPV } & \text { NPV } & \text { PLR } & \text { NLR } & \text { Kappa }\end{array}$

Patients with normal serum transaminases $(n=107)$

FIB-4 cutoffs

\begin{tabular}{|c|c|c|c|c|c|c|c|c|c|c|c|}
\hline$>1.3$ & 70 & 79 & - & - & 0.304 & 0.214 & 0.471 & 0.904 & 3.246 & 0.387 & 0.410 \\
\hline$>2.67$ & 13 & 99 & - & - & 0.870 & 0.012 & 0.750 & 0.806 & 10.957 & 0.880 & 0.169 \\
\hline$>1.45$ & 52 & 81 & - & - & 0.478 & 0.190 & 0.429 & 0.861 & 2.739 & 0.591 & 0.307 \\
\hline$>3.25$ & 0 & 100 & - & - & 0.957 & 0 & 1 & 0.792 & - & 0.792 & 0.067 \\
\hline \multicolumn{12}{|c|}{ Optimal FIB-4 cutoff } \\
\hline$>1.3$ & 70 & 79 & 0.757 & $0.644-0.870$ & 0.304 & 0.214 & 0.471 & 0.904 & 3.246 & 0.387 & 0.410 \\
\hline \multicolumn{2}{|c|}{ FIB-4 cutoffs } & & & & & & & & & & \\
\hline$>2.67$ & 18 & 97 & - & - & 0.821 & 0.026 & 0.667 & 0.805 & 6.955 & 0.843 & 0.207 \\
\hline$>1.45$ & 54 & 77 & - & - & 0.463 & 0.232 & 0.400 & 0.852 & 2.318 & 0.602 & 0.272 \\
\hline$>3.25$ & 15 & 98 & - & - & 0.851 & 0.021 & 0.667 & 0.800 & 6.955 & 0.869 & 0.177 \\
\hline \multicolumn{12}{|c|}{ Optimal FIB-4 cutoff } \\
\hline
\end{tabular}

AUC: Area under curve; Cl: Confidence interval; FN: False negative; FP: False positive; PPV: Positive predictive value; NPV: Negative predictive values; PLR: Positive likelihood ratio; NLR: Negative likelihood ratio.

Table 4. Diagnostic performance of the NFS in patients with normal $(n=107)$ and elevated $(n=300)$ serum transaminases in assessment of advanced fibrosis

\begin{tabular}{|c|c|c|c|c|c|c|c|c|c|c|c|}
\hline & Sensitivity (\%) & Specificity (\%) & AUC & $95 \% \mathrm{Cl}$ & FN & FP & PPV & NPV & PLR & NLR & Kappa \\
\hline \multicolumn{12}{|c|}{ Patients with normal serum transaminases $(n=107)$} \\
\hline \multicolumn{12}{|c|}{ NFS cutoffs } \\
\hline$>0.676$ & 26 & 91 & - & - & 0.739 & 0.095 & 0.429 & 0.817 & 2.739 & 0.817 & 0.193 \\
\hline \multicolumn{12}{|c|}{ Optimal NFS cutoff } \\
\hline$>-1.455$ & 76 & 61 & - & - & 0.239 & 0.395 & 0.357 & 0.898 & 1.928 & 0.395 & 0.261 \\
\hline$>0.676$ & 16 & 99 & - & - & 0.836 & 0.013 & 0.786 & 0.804 & 12.751 & 0.847 & 0.211 \\
\hline \multicolumn{12}{|c|}{ Optimal NFS cutoff } \\
\hline$>-1.483$ & 79 & 59 & 0.734 & $0.668-0.799$ & 0.209 & 0.408 & 0.358 & 0.908 & 1.940 & 0.353 & 0.268 \\
\hline
\end{tabular}

AUC: Area under curve; Cl: Confidence interval; FN: False negative; FP: False positive; PPV: Positive predictive value; NPV: Negative predictive values; PLR: Positive likelihood ratio; NLR: Negative likelihood ratio.

with the cutoff reported in the literature. ${ }^{[12]}$ The best agreement was also detected at this cutoff for patients with normal serum transaminases (kappa $=0.410)$, whereas a FIB-4 cutoff of $>1.2$ showed the best agreement in patients with elevated serum transaminases (kappa=0.269). Additionally, for both FIB-4 and NFS, we concluded that noninvasive tests were useful in exclusion of advanced fibrosis rather than identification of it as recommended in the guideline of European Association for the Study of the Liver, ${ }^{[3]}$ but in contrast with the guideline of American Association for the Study of Liver Diseases. ${ }^{[9]}$

The prevalence of NASH with advanced fibrosis is not well defined, and current estimates tend to underestimate the true prevalence given the assessment of advanced fibrosis was estimated with noninvasive methods rather than using reference standard. ${ }^{[18-20]}$ However, from Turkey, advanced fibrosis was reported to affect a substantial number of patients (17.3\%) with biopsy-proven NAFLD, which also shows the poor diagnostic accuracy of noninvasive tests in the identification of advanced fibrosis. ${ }^{[14]}$ Therefore, liver biopsy remains still the reference standard for the identification of histological features, ${ }^{[21,22]}$ especially the detection of advanced fibrosis represents a clinical significance, which is associated with a higher risk of liver-related morbidity and mortality. Therefore, it becomes a major focus for drug trials among patients with NASH. ${ }^{[23-27]}$ In other respects, it is of critical importance 
to exclude those patients with advanced fibrosis accurately to avoid unnecessary liver biopsy. ${ }^{[28]}$

Although both FIB-4 and NFS showed similar diagnostic performance concerning the exclusion of advanced fibrosis, FIB-4 can be used over NFS because of its slightly better performance and easier application using only four parameters in clinical practice, which was also reported previously. ${ }^{[29]}$ Additionally, following first-line triaging of NAFLD patients with advanced fibrosis with FIB-4 and NFS, performing Fibroscan to those patients were found to increase diagnostic accuracy, ${ }^{[30]}$ which was considered as a useful diagnostic tool in screening liver involvement in patients with NAFLD. ${ }^{[31]}$ In this scenario, patients who classified as low risk for advanced fibrosis must be managed in primary care, whereas patients with high risk should be directly referred to secondary care. However, patients who were classified as indeterminate risk according to FIB-4 or NFS should undergo Fibroscan examination. ${ }^{[32]}$

Our study has several limitations. Firstly, we performed this study focusing only on FIB-4 and NFS without investigating other noninvasive scores. ${ }^{[29]}$ Secondly, we defined normal serum transaminase levels according to our laboratory, which may not be compatible with other laboratories' diagnostic values.

\section{Conclusion}

In conclusion, FIB-4 and NFS showed acceptable diagnostic performance in the exclusion of advanced fibrosis rather than detection of it. Among those, FIB-4 can be the noninvasive diagnostic test of choice in clinical routine, considering its easier calculation with fewer parameters.

Ethics Committee Approval: This study was conducted in adherence to the Helsinki Declaration and was approved by the Marmara University ethics committee (date: 01.11.2019, number: 09.2019.944).

Peer-review: Externally peer-reviewed.

Author Contributions: EK, FE, YY: Conception and design of the study; EK, AB: Data acquisition; EK, AB, YY: Statistical analysis; EK, AB, FE, YY: Data analysis and drafting of the manuscript. All authors critically revised the manuscript and approved the final version.

Conflict of Interest: The authors have no conflict of interest to declare.

Financial Disclosure: This work has been supported by Marmara University Scientific Research Projects Coordination Unit under grant number: SAG-CTUP-150218-0033.

\section{References}

1. Sayiner M, Koenig A, Henry L, Younossi ZM. Epidemiology of nonalcoholic fatty liver disease and nonalcoholic steatohepatitis in the United States and the rest of the world. Clin Liver Dis 2016;20(2):205-214.

2. Younossi Z, Tacke F, Arrese M, Chander Sharma B, Mostafa I, Bugianesi E, et al. Global Perspectives on Non-alcoholic Fatty Liver Disease and Nonalcoholic Steatohepatitis. Hepatology 2019;69(6):2672-2682.

3. European Association for the Study of the Liver (EASL); European Association for the Study of Diabetes (EASD); European Association for the Study of Obesity (EASO). EASL-EASD-EASO Clinical Practice Guidelines for the management of non-alcoholic fatty liver disease. J Hepatol 2016;64(6):1388-1402.

4. Kaya E, Yılmaz Y. Non-alcoholic fatty liver disease: A growing public health problem in Turkey. Turk J Gastroenterol 2019;30(10):865-871.

5. Yilmaz Y. Review article: is non-alcoholic fatty liver disease a spectrum, or are steatosis and non-alcoholic steatohepatitis distinct conditions? Aliment Pharmacol Ther 2012;36(9):815-823
6. Bedossa P. Diagnosis of non-alcoholic fatty liver disease/non-alcoholic steatohepatitis: Why liver biopsy is essential. Liver Int 2018;38 (Supp 1):6466.

7. Younossi Z, Stepanova M, Ong JP, Jacobson IM, Bugianesi E, Duseja A, et al. Nonalcoholic Steatohepatitis Is the Fastest Growing Cause of Hepatocellular Carcinoma in Liver Transplant Candidates. Clin Gastroenterol Hepatol 2019;17(4):748-755.e3.

8. Brunt EM. Nonalcoholic fatty liver disease and the ongoing role of liver biopsy evaluation. Hepatol Commun 2017;1(5):370-378.

9. Chalasani N, Younossi Z, Lavine JE, Charlton M, Cusi K, Rinella M, et al. The diagnosis and management of nonalcoholic fatty liver disease: Practice guidance from the American Association for the Study of Liver Diseases. Hepatology 2018;67(1):328-357.

10. Yilmaz Y. Biomarkers for early detection of non-alcoholic steatohepatitis: implications for drug development and clinical trials. Curr Drug Targets 2013;14(11):1357-1366.

11. Stefan N, Häring HU, Cusi K. Non-alcoholic fatty liver disease: causes, diagnosis, cardiometabolic consequences, and treatment strategies. Lancet Diabetes Endocrinol 2019;7(4):313-324.

12. Shah AG, Lydecker A, Murray K, Tetri BN, Contos MJ, Sanyal AJ; Nash Clinical Research Network. Comparison of noninvasive markers of fibrosis in patients with nonalcoholic fatty liver disease. Clin Gastroenterol Hepatol 2009; 7(10):1104-1012.

13. Angulo P, Hui JM, Marchesini G, Bugianesi E, George J, Farrell GC, et al. The NAFLD fibrosis score: a noninvasive system that identifies liver fibrosis in patients with NAFLD. Hepatology 2007;45(4):846-854.

14. Yılmaz Y, Kanı HT, Demirtaş CÖ, Kaya E, Sapmaz AF, Qutranji L, et al. Growing burden of nonalcoholic fatty liver disease in Turkey: A single-center experience. Turk J Gastroenterol 2019;30(10):892-898.

15. Yilmaz Y, Eren F. Serum biomarkers of fibrosis and extracellular matrix remodeling in patients with nonalcoholic fatty liver disease: association with liver histology. Eur J Gastroenterol Hepatol 2019;31(1):43-46.

16. Subasi CF, Aykut UE, Yilmaz Y. Comparison of noninvasive scores for the detection of advanced fibrosis in patients with nonalcoholic fatty liver disease. Eur J Gastroenterol Hepatol 2015;27(2):137-141.

17. Sterling RK, Lissen E, Clumeck N, Sola R, Correa MC, Montaner J, et $\mathrm{al}$; APRICOT Clinical Investigators. Development of a simple noninvasive index to predict significant fibrosis patients with $\mathrm{HIV} / \mathrm{HCV}$ co-infection. Hepatology 2006;43(6):1317-1325.

18. Richards JA. Remote Sensing Digital Image Analysis: An Introduction. $5^{\text {th }}$ ed. Berlin: Springer Berlin Heidelberg; 2013.

19. Wong RJ, Liu B, Bhuket T. Significant burden of nonalcoholic fatty liver disease with advanced fibrosis in the US: a cross-sectional analysis of 20112014 National Health and Nutrition Examination Survey. Aliment Pharmacol Ther 2017;46(10):974-980.

20. Kabbany MN, Conjeevaram Selvakumar PK, Watt K, Lopez R, Akras Z, Zein N, et al. Prevalence of Nonalcoholic Steatohepatitis-Associated Cirrhosis in the United States: An Analysis of National Health and Nutrition Examination Survey Data. Am J Gastroenterol 2017;112(4):581-587.

21. Tsochatzis EA, Newsome PN. Non-alcoholic fatty liver disease and the interface between primary and secondary care. Lancet Gastroenterol Hepatol 2018;3(7):509-517.

22. Bril F, Cusi K. Nonalcoholic Fatty Liver Disease: The New Complication of Type 2 Diabetes Mellitus. Endocrinol Metab Clin North Am 2016;45(4):765-781.

23. Adams LA. End-points for drug treatment in NASH. Hepatol Int 2019;13(3):253-258.

24. Angulo P, Kleiner DE, Dam-Larsen S, Adams LA, Bjornsson ES, Charatcharoenwitthaya $\mathrm{P}$, et al. Liver fibrosis, but no other histologic features, is associated with long-term outcomes of patients with nonalcoholic fatty liver disease. Gastroenterology 2015;149(2):389-397.e10.

25. Ekstedt M, Hagström H, Nasr P, Fredrikson M, Stål P, Kechagias S, et al. 
Fibrosis stage is the strongest predictor for disease-specific mortality in NAFLD after up to 33 years of follow-up. Hepatology 2015;61(5):15471554.

26. Yilmaz Y, Younossi ZM. Obesity-associated nonalcoholic fatty liver disease. Clin Liver Dis 2014;18(1):19-31.

27. Yilmaz Y, Ulukaya E. Toward a biochemical diagnosis of NASH: insights from pathophysiology for distinguishing simple steatosis from steatohepatitis. Curr Med Chem 2011;18(5):725-732.

28. Kaya E, Bakir A, Kani HT, Demirtas CO, Keklikkiran C, Yilmaz Y. Simple non-invasive scores are clinically useful to exclude - rather than predict - advanced fibrosis: A study in Turkish patients with biopsy-proven nonalcoholic fatty liver disease. Gut Liver. 2019 Sep 19. [Epub ahead of print].

29. Cheah MC, McCullough AJ, Goh GB. Current modalities of fibrosis as- sessment in non-alcoholic fatty liver disease. J Clin Transl Hepatol 2017;5(3):261-271.

30. Petta S, Wong VW, Cammà C, Hiriart JB, Wong GL, Vergniol J, et al. Serial combination of non-invasive tools improves the diagnostic accuracy of severe liver fibrosis in patients with NAFLD. Aliment Pharmacol Ther 2017;46(6):617-627.

31. Demir M, Deyneli O, Y1lmaz Y. Screening for hepatic fibrosis and steatosis in Turkish patients with type 2 diabetes mellitus: A transient elastography study. Turk J Gastroenterol 2019;30(3):266-270.

32. Jafarov F, Kaya E, Bakir A, Eren F, Yilmaz Y. The diagnostic utility of FIB4 or NFS combined with liver stiffness measurement by Fibroscan in assessment of advanced liver fibrosis: A biopsy-proven NAFLD study. Eur J Gastroenterol Hepatol 2019 Oct 22 [Epub ahead of print]. 